\title{
Branching Out with Coevolutionary Trees
}

\author{
Kari A. Segraves
}

Published online: 7 January 2010

(C) Springer Science+Business Media, LLC 2009

\begin{abstract}
Coevolution (reciprocal evolutionary change in interacting species) is posited as a major mechanism that creates new species. A challenge has been to understand how coevolution has shaped the patterns of relatedness of interacting species and the traits involved in the interaction. Ongoing advances in the field of molecular phylogenetics have opened exciting avenues to examine both ancient and recent coevolutionary processes. Using plant-insect interactions as examples, I review the predictions of a number of coevolutionary models.
\end{abstract}

Keywords Coevolution - Escape and radiate .

Geographic mosaic theory - Molecular clock · Phylogenetics . Plant-insect interactions

Species interactions come in a variety of forms from loose, intermittent associations to those that are surprisingly specialized and intimate. For many species interactions, evolutionary change has clearly shaped one or both partners and in some cases has created fantastic adaptations. Some of the most impressive examples of adaptation come from highly specialized interactions. For instance, the interaction between yuccas and their pollinating moths is well known for its extreme specialization. Most species of yucca are only pollinated by a single species of moth, and the moths have extraordinary traits and behaviors to achieve pollination.

Prior to pollination, a female yucca moth lays an egg inside of the yucca flower using a structure called the ovipositor (Fig. 1). The ovipositors of yucca moths are

K. A. Segraves $(\bowtie)$

Department of Biology, Syracuse University,

107 College Place,

Syracuse, NY 13244, USA

e-mail: ksegrave@syr.edu specially modified to allow them to inject eggs into plant tissue hypodermically, and the placement of the eggs can be very precise. After ovipositing, a female will immediately climb to the top of the flower and actively pollinate using a small amount of pollen from the pollen ball that she stores stuck on the underneath side of her head. Even though there are thousands of pollination mutualisms, active pollination has only evolved four times. In yucca moths, females accomplish pollination by using specialized mouthparts called maxillary tentacles. These structures are not found in any other lepidopteran and are only used to collect and deposit pollen. The moths actively pollinate to ensure a food source for their developing offspring. The larvae feed within the yucca fruit, consuming a portion of the seeds. In the end, the plant gains pollination at the cost of losing a few seeds, and the moth earns a well protected site to rear their offspring. This interaction has become so specialized that neither the plant nor moth could survive without its partner.

The purposeful action of the yucca moths piqued my interest in how such spectacular behaviors and specialization could evolve. The moths have clearly gained a number of traits through evolutionary adaptation due to their interaction with yuccas, including the ability and sensory equipment to find host plants, specialized structures for pollinating and laying eggs into yucca flowers, and the associated behaviors required to accomplish pollination and oviposition (Pellmyr 2003). Likewise, the plants have also undergone similar evolutionary changes such as an unusually constant floral fragrance (Svensson et al. 2005), flowers that open at night, and the loss of nectar production. Traits associated with the interaction between plant and moth may have been shaped by reciprocal evolutionary pressures caused by the interaction itself. As the moths gained the structures and behaviors allowing them to become excellent pollinators, evolution would have favored 


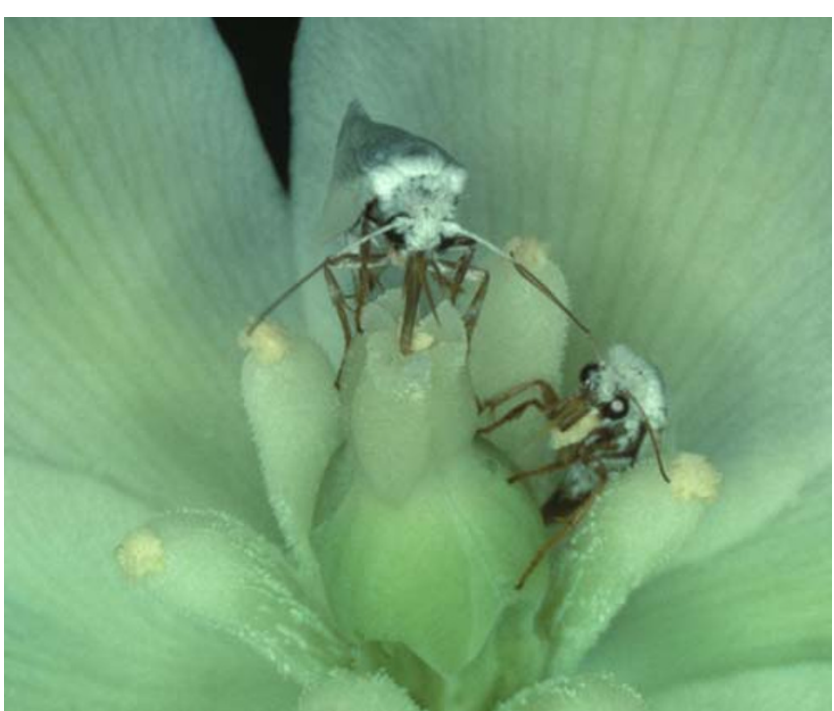

Fig. 1 Two yucca moth females in a yucca flower. The female on the right is laying an egg and clearly shows the pollen ball on the underside of her head. The female on the left is actively pollinating with her maxillary tentacles. Photograph courtesy of Olle Pellmyr

plants that saved energy by the cessation of nectar production and enhanced attraction mechanisms.

Coevolution, defined as reciprocal evolutionary change between interacting species, is thought to be one of the major evolutionary forces that generates new species (Thompson 1994). Examine any ecological community and you will find a multitude of species interactions, with a given organism interacting with several to hundreds of species over its life span. Some of these interactions will be brief and are unlikely to affect the evolution of a species, whereas other interactions may drastically alter the evolutionary pathways of the species involved. Some of these interactions have been evolving for millions of years, and the ever-improving tools for studying these long histories are helping us to unravel the large-scale patterns generated by the coevolutionary process.

\section{Phylogenetics: A Tool for Coevolutionary Studies}

By using genes that evolve at different rates and rapidly improving statistical tools, the field of molecular phylogenetics is refining our understanding of how species are related to each other. These phylogenetic frameworks are providing evidence of how traits of species have been modified as natural selection has continued to reshape them over millions of years. As we have learned more about the coevolutionary process, it has become clear that a comprehensive study of coevolution is incomplete without a phylogenetic framework in the same way that a puzzle lacking pieces is incomplete. Although assembling the puzzle is possible even when many pieces are missing, you may get the wrong impression about what the puzzle depicts. Likewise, coevolutionary studies of organisms that lack phylogenies may present a misleading picture.

The ultimate goal of phylogenetics is to create a phylogeny - a hypothesis depicting the evolutionary relationships of species. These phylogenies or "trees" are presented as branching diagrams with species placed at the tips of the branches and ancestors represented by the nodes or splits in the diagram. Species that share a recent ancestor are thought to be more closely related than species that share a more distant ancestor. Thus, trees provide information about the pattern of speciation (the formation of new species) and, in turn, this pattern can be used to infer how coevolution has shaped the evolutionary paths of species.

Phylogenies are being used in coevolutionary studies in two major ways. One is to examine the evolutionary histories of interacting species in order to create phylogenies for each species and compare their branching patterns. For example, we might expect that intimately associated species such as a parasite and its host would have similar branching patterns (Fig. 2a). If the parasites are vertically transmitted (passed from parent to offspring), we would expect the parasites to split into new species each time the hosts underwent speciation. In contrast, more loosely associated species may have quite different patterns of speciation, indicating 'jumps' to unrelated hosts (Fig. 2b). Making these types of comparisons of evolutionary trees can provide useful information about the factors involved in speciation and the extent to which coevolution has played a role in generating that diversity.

The other major use of phylogenetic approaches is to study how coevolved traits have changed through time. Although trees can be constructed using a number of different types of data, DNA sequence data are now most commonly used. One advantage to using DNA-based data is that it provides an assessment of the relationships among organisms independent of the phenotype, or outward appearance. This makes it possible to trace the evolutionary path of a particular trait. For instance, we could test whether maxillary tentacles and active pollination evolved once or repeatedly among the yucca moths (Fig. 3). Thus, by using a phylogenetic framework, we can learn a great deal about the lability of trait evolution and the origins of coevolved traits.

\section{Inferring Coevolutionary History-Phylogenetic Patterns at the Species Level}

A longstanding misconception about coevolution is that the speciation patterns of coevolving species should occur in parallel. That is, that we should always expect to find cospeciation or matching trees between coevolving species 


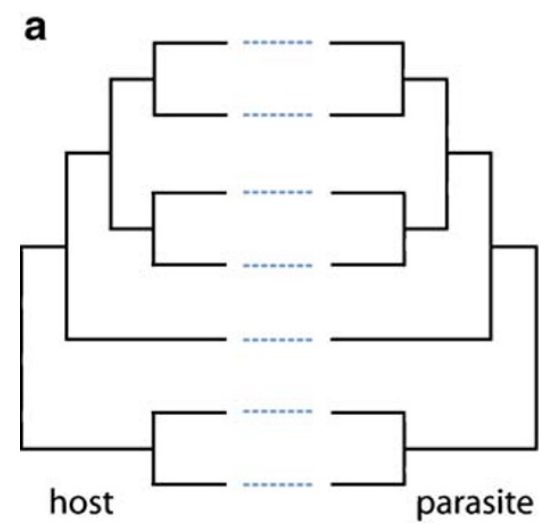

Fig. 2 Comparison of host and parasite phylogenetic trees. The dotted lines indicate host associations of the parasites. a Matching speciation events between host and parasite show a strict pattern of cospeciation.

(Fig. 2a). Although this is one potential phylogenetic result, cospeciation or lack thereof is not evidence for or against coevolution. Imagine a scenario where a verticallytransmitted symbiont (i.e., one passed generation after generation directly from a mother to her offspring) depends

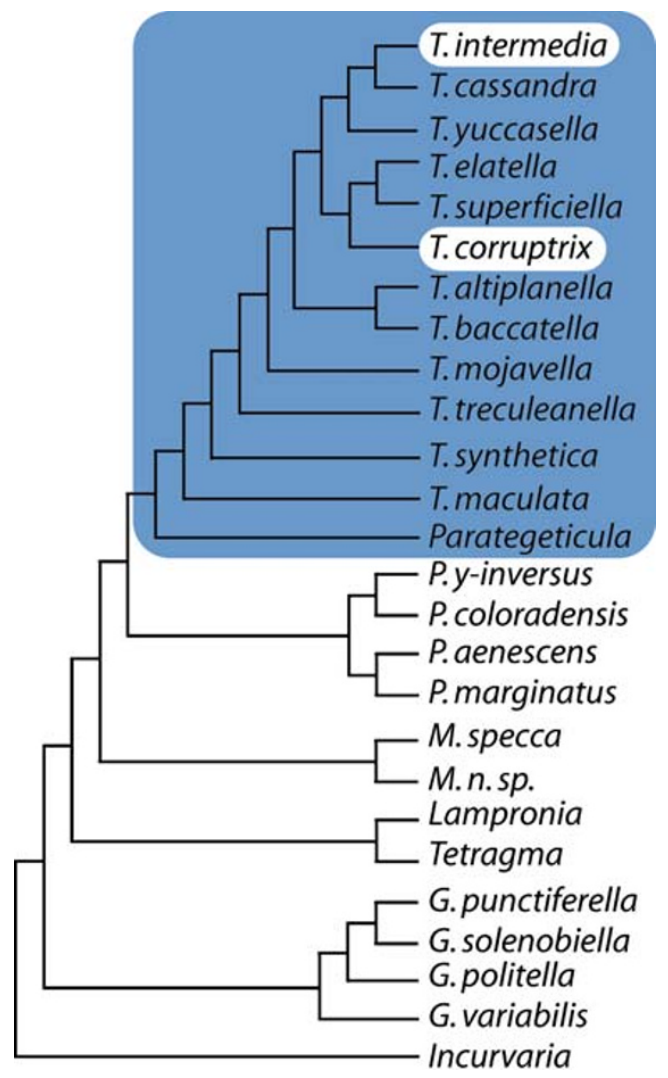

Fig. 3 Molecular phylogeny of the yucca moths and their close relatives based on mitochondrial DNA sequences. The large box indicates the evolution of active pollination and the maxillary tentacles. These traits evolved once and were subsequently lost in the two indicated lineages ( $T$. intermedia and $T$. corruptrix). Redrawn from Pellmyr and Leebens-Mack (1999). Abbreviations: T. Tegeticula, P. Prodoxus, M. Mesepiola, G. Greya

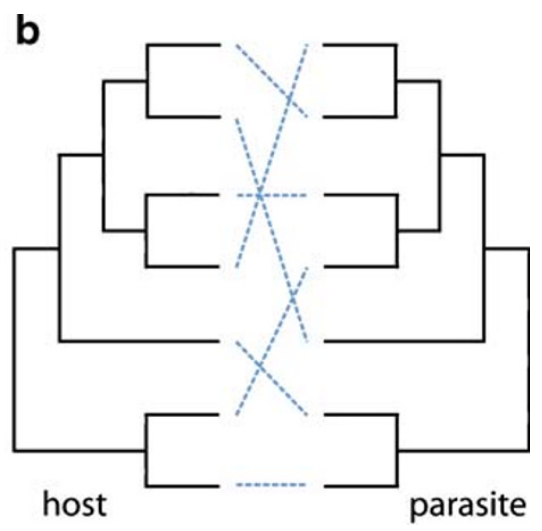

b Host switches to distant relatives are shown by the crossing lines. Some parasites use closely related hosts, but most have jumped to a distant relative

on a host for its survival and reproduction. The presence of the symbiont has no impact on the host; thus, the host will not have an evolutionary response (although the symbiont is likely to have evolved adaptations to the host). Due to vertical transmission of the symbiont, each time the host lineage splits into new species, in time, so does the symbiont. Clearly, there is no reciprocal evolutionary change, and so the perfectly matched phylogenies will have occurred without coevolution. Vertical transmission also occurs in many hostparasite interactions; thus, cospeciation in most host-parasite interactions is probably caused by this mode of transmission and should not be interpreted as evidence for coevolution.

Cospeciation may also occur independently of coevolution when speciation is caused via the separation of populations by geographic barriers such as mountain ranges (Page 1994; Roderick 1997). Such "vicariant events" lead to speciation by physically separating populations long enough for reproductive isolation to occur. Vicariance is likely the source of parallel phylogenies between taxa such as Cryptocercus cockroaches and the endosymbiotic bacteria that live in their fat bodies. Comparisons of these phylogenies have shown that for each speciation event in the cockroach, there was a matching split in the bacterial tree (Clark et al. 2001). Furthermore, speciation has been concordant with major vicariant events, suggesting an initial split into two lineages - one occupying Asia and another moving into North America. These lineages became separated with the breakup of the continents and the loss of land bridges. Upon entering North America, the incursion of a large inland sea further broke the North American lineage into eastern and western species. Although the bacteria and cockroaches may be coevolving, the pattern of cospeciation is most likely caused by geological and climatic events.

Cospeciation is not a prediction of coevolution, but there are some circumstances where we would expect to find matching patterns of speciation. Intimately associated 
species such as vertically transmitted symbionts and their hosts may cospeciate by default due to the mode of transmission, especially if there is low probability for horizontal transfer to a new host species. We would also predict highly specialized, coevolved interactions to be good candidates for parallel speciation. For similar reasons, the intimacy of the association may prevent species from making phylogenetically drastic switches to new partners. Interacting with a distant relative of your partner may not be an option if coevolution has created incompatibilities in specialized traits required for the interaction. For instance, if laying eggs into yucca flowers requires a particular complement of ovipositor traits and moth behaviors, we might predict to find host switches only between closely related yuccas. Initial analyses of yucca and yucca moth phylogenies suggest that the trees are somewhat congruent, although there have been at least two instances of moths switching hosts to quite distantly related yuccas (Pellmyr 2003; Althoff et al., unpublished). The interaction between yuccas and yucca moths is clearly one that is highly specialized and coevolved, yet we do not find a phylogenetic pattern of perfect matching between plant and insect.

Aside from yuccas and their moths, there are only three known cases of active pollination where the females purposefully pollinate the plant: senita cacti and senita moths, figs and fig wasps, and Glochidion trees and Epicephala moths. Unfortunately, cospeciation analysis is precluded for the senita interaction as there is only one moth species that pollinates a single plant species (Holland and Fleming 1999). In contrast, figs are extremely species-rich, including over 700 species worldwide. They are pollinated by miniscule wasps that must force their way through a tiny opening into the enclosed cavity of the fig inflorescence. Upon entering the inflorescence, the female will pollinate and lay eggs in some of the flowers. The offspring feed on the endosperm (tissue surrounding the embryo that is used for nutritional storage) of a single seed within the developing fig. Upon emerging as an adult, a female wasp mates, collects pollen, and exits the fig through a hole that the males chew for her. In addition to the pollinators, there are also parasitic wasps that use long ovipositors to pierce through the wall of the inflorescence and lay eggs without pollinating. Comparisons of the phylogenies of figs with both of these types of wasps show some degree of cospeciation between figs and their pollinators (e.g., LopezVaamonde et al. 2001; Jackson 2004; Jousselin et al. 2008) whereas comparisons of figs and the parasitic wasps did not show cospeciation (Weiblen and Bush 2002, but see Jousselin et al. 2008). This suggests that the intimacy of the interaction with pollinators is greater than that of parasites. Although cospeciation analyses have shown a significant level of cospeciation with plants and pollinators, much like the yucca-yucca moth example, host switches to more distantly related figs are quite common (Machado et al. 2005).
The most recently discovered example of active pollination is surprisingly similar to the yucca-yucca moth interaction. Much like yucca moths, female Epicephala moths use mouthparts to collect and deposit the pollen of Glochidion trees. After pollinating, they lay an egg inside of the flower, and the offspring feed on developing seeds. Cospeciation analysis shows that the plant and moth trees are more similar than expected based on chance, although there are a number of instances of host shifts (Kawakita et al. 2004). Consequently, the overall patterns for the Glochidion-Epicephala, fig-fig wasp, and yucca-yucca moth interactions largely follow the prediction of parallel speciation, but the matching of speciation events is far from perfect. Host shifts are a common theme and do not seem to be limited by the host's degree of relatedness. Perhaps these patterns are a result of coevolutionary processes driving the formation of new species in both the plant and insect lineages. Determining whether this is the case requires more information than simple comparisons of phylogenies.

As a case in point, consider the interaction between Bursera trees and leaf beetles in the genus Blepharida. The beetles are specialized herbivores of Bursera and both have evolved adaptations in response to the interaction (Becerra 1994). The plants have an intricate defense against the beetles - they pressurize their leaf veins with a sticky, toxic resin. When an unsuspecting herbivore severs one of these veins, it gets a squirt of gluey toxins that may be lethal or will at least render the insect unable to feed for some period of time. In response, the beetles have evolved specialized behaviors that allow them to avoid this "squirt gun defense". They nip at the main leaf vein, gradually draining the pressure of the toxins. Once the pressure is relieved, they are free to feed on the leaf. In contrast with the pollination interactions discussed above, a comparison of the pattern of speciation in beetles and host plants shows that there is little evidence for cospeciation and that host shifts have occurred frequently (Becerra 1997). If the beetle phylogeny is compared to plant chemical defenses, however, the repeated host shifts make good sense: beetles are likely to shift between hosts that are chemically similar. Without the additional information about the host plants, the cospeciation results could be misinterpreted as evidence for a diffuse interaction rather than a tightly coevolved system where beetle speciation is strongly limited by host plant chemistry. As a consequence, understanding the underlying ecology of the interaction is an essential aspect of studying coevolution.

\section{Phylogenetic Predictions of Escape and Radiate Coevolution}

In addition to illustrating the importance of linking ecology with phylogenetic data, the Bursera-Blepharida example 
also demonstrates that parallel speciation is a poor predictor of some coevolutionary scenarios. In fact, specific types of coevolutionary processes may predict alternative patterns to cospeciation. One such process is that of escape and radiate coevolution. First introduced by Ehrlich and Raven (1964), this hypothesis proposed a mechanism of speciation for two incredibly species-rich groups of organisms: plants and the insects that feed on them. The idea is that an evolutionary arms race between plants and herbivorous insects drives reciprocal bursts of speciation in each group. The plants evolve a defense to the herbivores which prevents the insects from being able to feed on the plants. This defense could take a number of forms including the squirt gun defense of Bursera or another toxic chemical defense. Escape from herbivory opens an ecological opportunity that allows the newly defended plants to undergo rapid and repeated speciation events. These rapid speciation events are often referred to as species radiations. Eventually, the insects evolve a counter-defense to the plant defense. The insects can then feed on the plants, opening an ecological opportunity for them to diversify onto the new lineage of plants. This process may repeat itself as the defenses and counter-defenses escalate.

Escape and radiate coevolution, then, offers several specific phylogenetic predictions (Fig. 4). First, this hypothesis predicts that coevolved plants and insects should be more species rich than closely related plants and insects that are not coevolving. Second, when the evolution of a novel defense or counter-defense provides ecological opportunity for new species to form, the tempo of speciation is quick. Therefore, the coevolving plants and insects should have star-like phylogenies - trees that have short internal branch lengths typical of rapid speciation

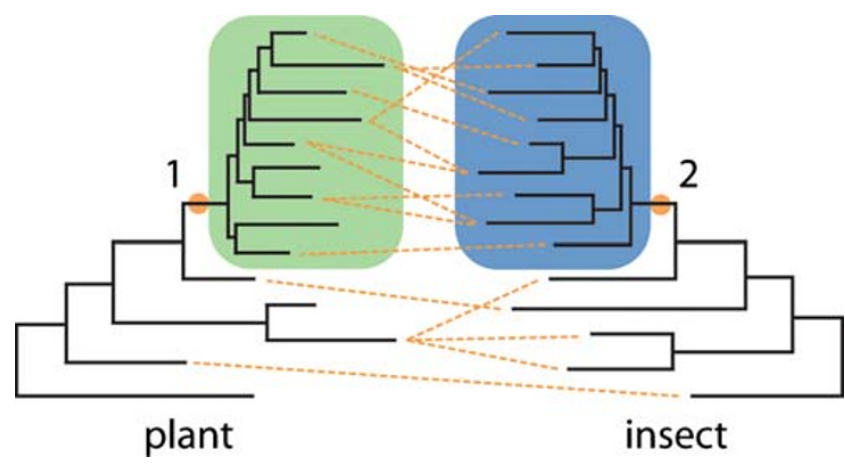

Fig. 4 Comparison of plant and insect phylogenies consistent with escape and radiate coevolution. At time 1, the plant evolves a defense trait (orange circle) and then radiates into a number of new species that have the defense trait (green box). At a point later in time (time 2), the insect evolves a counter-defense trait (orange circle) and then radiates onto the newly evolved plant lineage. All insect species in the radiation share the counter-defense trait (blue box). Colonization of the plants does not follow a pattern of cospeciation (dotted lines) events. These bursts of speciation should be associated with the evolution of a novel defense or counter-defense trait. Third, the pattern of colonization of the insects on the hosts should be independent of the evolutionary history of the plant. Because the plants have evolved in the absence of herbivory, there is no reason to expect that the insects will colonize the plants beginning with the oldest lineage and proceeding to the youngest. As a consequence, we should predict that there will be no congruence between plant and insect phylogenies. Fourth, escape and radiate predicts a time lag between the plant and insect radiations. The plants evolve a defense first and radiate in the absence of herbivory. After a lag period, the insects evolve a counterdefense and radiate. Although escape and radiate coevolution is thought to be an important process contributing to species diversity, phylogenetic evidence supporting these predictions is surprisingly scant (Futuyma and Agrawal 2009). There are, however, a few studies that provide clues about the validity of the escape and radiate model.

For instance, the first prediction of enhanced species richness in coevolving lineages was evaluated in the Apiaceae, a family of plants that contain toxic compounds called coumarins. Berenbaum (1983) suggested that the plants have undergone an escalation of defenses to herbivory, evolving more and more toxic coumarins. Lineages that have these compounds have greater species diversity, suggesting that escape from herbivory may have enhanced diversification. This seminal paper spurred an additional test in plants defended by latex and resin canals. Many plants store sticky and/or toxic fluids in specialized canals that release secretions when damaged by herbivores. Farrell et al. (1991) compared a diverse set of plant groups that had specific phylogenetic properties. They examined paired lineages that were each other's closest relatives (sister groups); one member of the pair was defended by latex and resin canals whereas the other member was not defended. In doing so, they had repeated tests across diverse taxa of whether evolving a defense trait enhances species richness. Thirteen of the 16 sister group comparisons had greater species richness in the plant lineages with resin and latex canals.

Coupled with the idea that escape and radiate creates species-rich groups is the prediction that the tempo of speciation should be quick and that the burst of speciation should occur concurrently with the evolution of a novel trait. Thus, the evolution of the trait should occur at the base of the species radiation (Fig. 4). Once the novel trait arises, the time between speciation events will occur more quickly, and may be so fast that more than two species arise simultaneously. Rapid speciation creates phylograms with short internal branches. A phylogram is a phylogeny where the branch lengths have been scaled to the amount of nucleotide change that has occurred along each branch 
(Figs. 4 and 5). The amount of nucleotide change correlates with time in the sense that the more time that passes prior to speciation, the longer the length of the branch. That is, longer intervals between speciation events allows more time for mutations to accumulate. Although a number of coevolved groups appear to have star-like phylogenies with short internal branches, formal statistical tests remain to be conducted.

Once the plant lineage has diversified in the absence of herbivory, the insects will evolve a counter-defense and will colonize the new plant species. Because the plants have already diversified, the order of colonization by the insects should be independent of the order of speciation in the plants. Thus, we would not expect to find cospeciation. This prediction, however, assumes that diversification in the plants was complete prior to colonization by the insects. If the insects colonize earlier during the plant radiation, then some degree of cospeciation may occur for the more recent lineages of plants and insects (Thompson 2005). Tests of this prediction will need to consider the timing of insect and plant diversifications to determine which parts of the trees should be discordant.

The fourth prediction of time lags between plant and insect radiations follows up on the idea that nucleotide change correlates with time. Phylogeneticists often use a molecular clock analysis to determine the approximate timing of events on trees. Zuckerkandl and Pauling (1965) recognized that mutations accumulated at regular intervals; thus, rates of mutation could be used to estimate time. For instance, if one mutation occurs every millionth generation, then two species that differed by three mutations would have split from each other approximately three million generations ago. This is a gross simplification of the actual statistical approaches and calibration procedures commonly used to estimate divergence times (Arbogast et al. 2002),

Fig. 5 Example of a phylogram. The timeline along the bottom shows how new lineages progressively appear in time. Species A and B diverged earlier in time than species $\mathrm{F}$ and $\mathrm{G}$. The arrows point to short internal branches indicative of a species radiation but serves to illustrate the general idea. A molecular clock, then, can be used to determine whether insect diversifications have lagged behind the radiation in their host plants. A survey examining 18 comparisons of plant and insect phylogenies showed either contemporaneous species radiations or that the insect lineages were younger than their associated host plant lineages (Winkler and Mitter 2008). Due to considerable error associated with estimating molecular clocks, some of the studies indicating synchronous evolution may actually have time lags (and vice versa for studies indicating lags). Taken at face value, however, these data are consistent with the prediction of escape and radiate coevolution and suggest that ecological opportunity created by a newly diversified plant lineage may be driving subsequent speciation in insect lineages.

\section{Ancient History-Phylogenetic Patterns Above the Species Level}

The same coevolutionary processes are acting at higher taxonomic levels; thus, we would predict the same phylogenetic patterns in these deep divergences. In these phylogenetic comparisons, rather than placing species on the tips of the tree, higher taxonomic units are used. For instance, if we were to conduct an analysis of tribes within a family, the tips of the branches are tribes which are typically defined by "representative" samples (e.g., a single species used to represent the group). Phylogenies created in this way will represent deeper evolutionary history and can be used to assess whether the coevolutionary patterns found at the species level translate into the formation of higher taxonomic units.

The same phylogenetic approaches used at the species level can be applied to examine whether the coevolutionary process generates deeper divergences. For instance, we can address whether the degree of intimacy of a coevolved interaction leads to cocladogenesis or parallel splitting of lineages in interacting taxa. In a study assessing the escape and radiate hypothesis, Janz and Nylin (1998) use a phylogenetic approach to show that closely related subfamilies of butterflies were more likely to feed on closely related groups of plants. Similar to the examples mentioned above, host shifts among more distantly related plant groups were also common. Although they demonstrated that at higher taxonomic levels there was a general tendency to shift among close relatives, this does not necessarily mean that cospeciation would be found at the species level.

Much like the host shifts of Blepharida beetles onto chemically similar host plants, host shifts in butterflies may be constrained by plant secondary chemistry. Shifts to chemically dissimilar hosts may be rare because the insects must first evolve a means of detoxifying the compounds. 
Just such a shift is proposed for the butterfly family Pieridae. A number of tribes within the subfamily Pierinae have switched from feeding on legumes to members of the Brassicales, a plant order heavily defended by glucosinolates. Phylogenetic analysis showed that the novel origin of a detoxification mechanism in the Pierinae allowed these butterflies to switch to Brassicales (Wheat et al. 2007). Furthermore, consistent with escape and radiate coevolution, the host shift and subsequent radiation lagged after diversification in the Brassicales, and the Pierinae are significantly more diverse than their closest relatives. Consequently, the coevolutionary process may lead to the genesis of higher taxa and deep divergence events.

\section{Recent Coevolution-Phylogenetic Patterns Below the Species Level}

Clearly, evidence is accumulating that coevolution contributes to macroevolutionary processes - evolution that leads to the formation of new species and taxonomic groups. These macroevolutionary patterns, however, are a consequence of evolution within species (Dobzhansky 1937). Microevolution, or the differentiation of populations within species, is the ultimate force that generates diversity. Population level divergence can lead to speciation in a number of ways. If populations are sufficiently subdivided so that there is no gene flow (movement of individuals and their genes among populations), random processes can lead to speciation. Natural selection can also cause speciation even when there is a fair degree of gene flow, especially in circumstances where geographically separated populations experience different selective forces. Teasing apart which of these scenarios is causing population divergence is an important first step in coevolutionary studies.

Phylogeographic analyses can be extremely useful in this regard. As the name suggests, phylogeography combines observations of the relatedness of individuals within species with the geographic distribution of those individuals (Avise 2000; Avise 2004). In this case, the tree has individuals on the tips of the branches and geographic regions are mapped onto the tree as traits. Usually, many individuals per population are sampled, which provides information on the historical patterns of movement and genetic exchange among populations. For example, the yucca moth Tegeticula intermedia has a broad geographic range, spanning from Florida and the southeastern USA westward into Texas, New Mexico, and Wyoming. A gap in the central USA roughly divides populations of $T$. intermedia into two groups - one on either side of the Mississippi River. Phylogeographic analysis showed that the split into eastern and western populations was associated with a substantial reduction in gene flow and that the most ancestral populations were in the western USA (Segraves and Pellmyr 2004). These data indicated that $T$. intermedia had originated in the west and migrated eastward and that subsequent geographic barriers had limited gene flow between the eastern and western populations. A number of other species show similar population subdivision on either side of the Mississippi River (e.g., Althoff and Pellmyr 2002; Calhoun 2002), suggesting that historical physical barriers to gene flow have been important in causing population divergence. Concordant patterns of population structure across a number of species provide strong evidence that geographic factors rather than coevolution, are shaping population level divergence.

One aspect of demonstrating coevolution, then, requires rejecting population subdivision caused by vicariance. Smith et al. (2008) took a comparative phylogeographic approach to do exactly this. They conducted phylogeographic analyses of Yucca brevifolia (Joshua tree) and its pollinator moths. Joshua tree is pollinated by two moth species that effectively split the range of the plants into eastern and western populations (Pellmyr and Segraves 2003). Historically, the range was divided by an incursion of the Sea of Cortez, which could have created the subdivision observed in modern populations. The plants, however, have substantial morphological differences between the eastern and western populations and some of these differences - particularly floral characters - may have been mediated by coevolution with the pollinators (Godsoe et al. 2008). Molecular clock estimates showed that the timing of divergence in Joshua tree was substantially earlier than in the moths, rejecting the hypothesis that a common vicariant event caused the divisions within these species. Coevolution may be changing patterns of gene flow and driving apart eastern and western populations.

Because population subdivision and gene flow are key components of the geographic mosaic theory of coevolution (GMTC), phylogeographic analysis will form the foundation of many studies of coevolution (Thompson 2005). The GMTC paints a dynamic view of coevolution across landscapes (Thompson, this issue). This theory predicts that geographic subdivision of populations creates a backdrop for variable selection that results in hotspots and coldspots-local sites where selection is either reciprocal or not. Gene flow among hotspots and coldspots will further change the local selection pressures. As a result, we can use phylogeographic analysis to examine population subdivision and gene flow to help identify probable sites where we expect to find hotspots and coldspots.

Under the GMTC, coevolving species should have some degree of phylogeographic structure where gene flow is restricted among populations or groups of populations. This restriction of gene flow creates the potential for the "mosaic" by allowing local populations to evolve indepen- 
dently of one another. Local adaptation could be one-sided or reciprocal. When gene flow is high, however, local adaptation is less likely to occur because different versions of the trait are constantly being added to the population (Althoff and Thompson 1999; Ridenhour and Nuismer 2007). Populations connected by high levels of gene flow are less likely to be locally adapted than isolated populations, and so we would predict to find coldspots in areas of high gene flow and hotspots in areas of restricted gene flow. Likewise, we would expect asymmetries in gene flow to create asymmetrical local adaptation; the partner with low levels of gene flow is locally adapted whereas the partner with high levels of gene flow is not (Ridenhour et al. 2007). Because phylogeographic analysis can provide key estimates of gene flow and connectedness among populations, I expect that it will be extremely useful in future studies of the GMTC.

\section{Conclusions}

One of the challenges in coevolutionary studies will be to determine the specific phylogenetic predictions under a model of coevolution and to determine what types of phylogenetic evidence support a coevolutionary scenario. Past misinterpretations of cospeciation warn us of the danger of comparing phylogenies alone. Clearly, the best studies will include lines of evidence from multiple sources - natural history, ecological work on the species involved, studies of natural selection on coevolved traits, examination of multiple populations through the geographic range of the interaction, and comparisons of closely related species involved in similar interactions. Therefore, meeting a phylogenetic expectation provides only one important clue about coevolution.

The future role of phylogenetics in coevolutionary studies is bright. We have already developed a number of phylogenetic predictions that are just beginning to provide powerful tests of coevolutionary hypotheses. For example, the paradigm of escape and radiate coevolution can be tested by looking for starbursts of speciation following the evolution of key defense and counter-defense traits. Phylogenetic analysis provides the flexibility to test hypotheses about the role of coevolution in ancient divergence events of higher taxa while at the same time allowing us to traverse all the way down the taxonomic hierarchy to examine how gene flow and population subdivision impact the geographic mosaic of coevolution. We are already following a number of exciting avenues of research by incorporating phylogenetics into studies of coevolution, and these will only grow as phylogenetic tools diversify.

Acknowledgments I thank David Althoff, Marv Druger, Rodrigo Medel, and John Thompson for comments on the manuscript and Olle Pellmyr for permission to use his photograph of the yucca moths.

\section{References}

Althoff D, Pellmyr O. Examining genetic structure in the bogus yucca moth: a sequential approach to phylogeography. Evolution. 2002;56:1632-43.

Althoff D, Thompson JN. Comparative geographic structures of two parasitoid-host interactions. Evolution. 1999;53:818-25.

Arbogast BS, Edwards SV, Wakeley J, Beerli P, Slowinski JB. Estimating divergence times from molecular data on phylogenetic and population genetic timescales. Annu Rev Ecol Syst. 2002;33:707-40.

Avise JC. Phylogeography: the history and formation of species. Cambridge: Harvard University Press; 2000.

Avise JC. Molecular markers, natural history, and evolution. Sunderland: Sinauer Associates, Inc.; 2004.

Becerra JX. Squirt-gun defense in Bursera and the chyrsomelid counterploy. Ecology. 1994;75:1991-6.

Becerra JX. Insects on plants: macroevolutionary chemical trends in host use. Science. 1997;276:253-6.

Berenbaum M. Coumarins and caterpillars: a case for coevolution. Evolution. 1983;37:163-79.

Calhoun JV. Sibling rivalry in Florida: the displacement of Pyrgus communis by Pyrgus ablescens (Hesperiidae). J Lepid Soc. 2002;56:98-103.

Clark JW, Hossain S, Burnside CA, Kambhampati S. Coevolution between a cockroach and its bacterial endosymbiont: a biogeographical perspective. Proc R Soc Lond B. 2001;268:393-8.

Dobzhansky T. Genetics and the origin of species. New York: Columbia University Press; 1937.

Ehrlich PR, Raven PH. Butterflies and plants: a study in coevolution. Evolution. 1964;18:586-608.

Farrell BD, Dussourd DE, Mitter C. Escalation of plant defense: do latex and resin canals spur plant diversification? Am Nat. 1991;138:881-900.

Futuyma DJ, Agrawal AA. Macroevolution and the biological diversity of plants and herbivores. Proc Natl Acad Sci USA. 2009;106:18054-61.

Godsoe WKW, Yoder JB, Smith CI, Pellmyr O. Coevolution and divergence in the Joshua tree/yucca moth mutualism. Am Nat. 2008; 171:816-23.

Holland JN, Fleming TH. Mutualistic interactions between Upiga virescens (Pyralidae), a pollinating seed-consumer, and Lophocereus schottii (Cactaceae). Ecology. 1999;80:2074-84.

Jackson AP. Cophylogeny of the Ficus microcosm. Biol Rev. 2004;79:751-68.

Janz N, Nylin S. Butterflies and plants: a phylogenetic study. Evolution. 1998;52:486-502.

Jousselin E, van Noort S, Berry V, Rasplus JY, Ronsted N, Erasmus JC, et al. One fig to bind them all: host conservatism in a fig wasp community unraveled by cospeciation analyses among pollinating and nonpollinating fig wasps. Evolution. 2008;62:1777-97.

Kawakita A, Takimura A, Terachi T, Sota T, Kato M. Cospeciation analysis of an obligate pollination mutualism: have Glochidion trees (Euphorbiaceae) and pollinating Epicephala moths (Gracillariidae) diversified in parallel? Evolution. 2004;58:2201-14.

Lopez-Vaamonde C, Rasplus JY, Weiblen GD, Cook JM. Molecular phylogenies of fig wasps: partial cocladogenesis of pollinators and parasites. Mol Phylogenet Evol. 2001;21:55-71.

Machado CA, Robbins N, Gilbert MTP, Herre EA. Critical review of host specificity and its coevolutionary implications in the fig/fig-wasp mutualism. Proc Natl Acad Sci USA. 2005;102:6558-65.

Page R. Maps between trees and cladistic analysis of historical associations among genes, organisms, and areas. Syst Biol. 1994;43:58-77.

Pellmyr O. Yuccas, yucca moths, and coevolution: a review. Ann Mo Bot Gard. 2003;90:35-55. 
Pellmyr O, Leebens-Mack J. Forty million years of mutualism: evidence for Eocene origin of the yucca-yucca moth association. Proc Natl Acad Sci USA. 1999;96:9178-83.

Pellmyr O, Segraves KA. Pollinator divergence within an obligate mutualism: two yucca moth species (Lepidoptera; Prodoxidae: Tegeticula) on the Joshua tree (Yucca brevifolia; Agavaceae). Ann Entomol Soc Am. 2003;96:716-22.

Ridenhour BJ, Nuismer SL. Polygenic traits and parasite local adaptation. Evolution. 2007;61:368-76.

Ridenhour BJ, Brodie Jr ED, Brodie III ED. Patterns of genetic differentiation in Thamnophis and Taricha from the Pacific Northwest. J Biogeogr. 2007;34:724-35.

Roderick GK. Herbivorous insects and the Hawaiian silversword alliance: coevolution or cospeciation? Pac Sci. 1997;51:440-9.

Segraves KA, Pellmyr O. Testing the 'Out of Florida' hypothesis on the origin of cheating in the yucca-yucca moth mutualism. Evolution. 2004;58:2266-79.

Smith CI, Godsoe WKW, Tank S, Yoder JB, Pellmyr O. Distinguishing coevolution from covicariance in an obligate pollination mutualism: asynchronous divergence in Joshua tree and its pollinators. Evolution. 2008;62:2676-87.
Svensson GP, Hickman MO, Bartram S, Boland W, Pellmyr O, Raguso RA. Chemistry and geographic variation of floral scent in Yucca filamentosa (Agavaceae). Am J Bot. 2005;92:1624-31.

Thompson JN. The coevolutionary process. Chicago: University of Chicago Press; 1994.

Thompson JN. The geographic mosaic of coevolution. Chicago: University of Chicago Press; 2005.

Weiblen G, Bush G. Speciation in fig pollinators and parasites. Mol Ecol. 2002;11:1573-8.

Wheat CW, Vogel $\mathrm{H}$, Wittstock U, Braby MF, Underwood D, Mitchell-Olds T. The genetic basis of a plant-insect coevolutionary key innovation. Proc Natl Acad Sci USA. 2007;104:2042731.

Winkler IS, Mitter C. The phylogenetic dimension of insect-plant interactions: a review of recent evidence. In: Tilmon KJ, editor. Specialization, speciation, and radiation: the evolutionary biology of herbivorous insects. Berkley: University of California Press; 2008. p. $240-63$.

Zuckerkandl E, Pauling LB. Evolutionary divergence and convergence in proteins. In: Bryson V, Vogel HJ, editors. Evolving genes and proteins. New York: Academic Press; 1965. p. 97-166. 